\section{UCDNN}

LIBRARY
University of Connecticut OpenCommons@UConn

Philosophy Articles

Philosophy Department

July 1998

\title{
What Sensory Signals are About
}

Crawford Elder

University of Connecticut Department of Philosophy, crawford.elder@uconn.edu

Follow this and additional works at: https://opencommons.uconn.edu/philo_articles

\section{Recommended Citation}

Elder, Crawford, "What Sensory Signals are About" (1998). Philosophy Articles. 4.

https://opencommons.uconn.edu/philo_articles/4 


\title{
What Sensory Signals are About
}

\author{
Crawford L. Elder
}

In 'Of Sensory Systems and the "Aboutness" of Mental States', Kathleen Akins (1996) argues against what she calls 'the traditional view' about sensory systems, according to which they are detectors of features in the environment outside the organism. As an antidote, she considers the case of thermoreception, a system whose sensors send signals about how things stand with themselves and their immediate dermal surround (a 'narcissistic' sensory system); and she closes by suggesting that the signals from many sensory systems may not in any familiar sense be about anything at all. Her presentation of the issues, however, overlooks resources available to 'the traditional view' —or so I shall argue.

Akins's own thumbnail sketch of what is wrong with the traditional view is that it asks, concerning a given sensory system, 'what is it detecting?', when we should instead be asking 'what is it doing?' (352). Her point is that on the traditional view the function of a sensory system—what it's 'for'-is to detect or indicate (values of) features of the outside environment. But at least on one version of the traditional view_namely Ruth Millikan's—-this would never be the sole or main proper function of a sensory system. (Akins does not list Millikan as a traditionalist, but Millikan fits squarely Akins's description of them, since she believes in a naturalistic theory of aboutness and thinks it should begin with the senses.) For Millikan (1989, 1993), the proper function of a sensory system is in the first instance enabling behavioural systems - in the simplest case, motor routines - to perform their proper function. This they do, roughly, by switching on and steering the behavioural routines. Where features of the outside environment come in is as Normal (= assumed-by-the-design) conditions for the successful performance of the sensory system's proper function. That is, the only strategy for switching on and steering that is simple enough for evolution to have hit upon it, and reliable enough for evolution to have liked it, is a strategy which gears the steering to (values of) features of the outside environment.

But as soon as one starts fleshing out the details of this story, one notices that they are probably quite different in the case of thermoreception from how they are with 'distance' senses such as vision and olfaction--a point which Akins overlooks. It's likely that a proper function (maybe the proper function) of 
the thermoreceptive system is to steer motor routines so as to ward off freezing and cooking of the skin. The only strategy for steering here which has the requisite combination of simplicity and reliability, would be one which gears the steering to the severity of the threat presently posed to the area of skin where the thermoreceptor itself is. This is exactly the feature to which thermoreceptors do respond, on Akins's own account. (Akins describes two different types of thermoreceptors, each characterized by two patterns of response to ambient temperature, but sums by saying 'the system as a whole constitutes one solution to man's various thermal needs--that he be warned when thermal damage is occurring or before it is likely to occur, when temperatures are likely to have specific consequences, and so on' (350).) And yes, severity of threat is only just barely a feature of the outside environment: it is a feature of the environment that touches the skin, and is very close to the thermoreceptors. It is entirely true then that the response-activity of thermoreceptors is 'narcissistic'.

Concerning other sensory systems and capacities, however, it is plausible to attribute proper functions of steering behaviour in more indirect ways. Shape recognition and distance perception are the most obvious examples, but I will focus on the more challenging examples of colour vision and olfaction. Colour vision, let us suppose, responds to something in the objects that surround the organism that can serve usefully to steer that organism's behaviour. But does it do this by indicating where particular colours are to be found? Does the system's testimony, that is, that colour $C$ is to be found not only here but there, or is present again just as it was a moment ago, attune the organism to useful objective samenesses in the organism's surroundings? The idea seems implausible, since a whole rabble of different surface reflectance profiles will present the same hue, and a coloured light of single wavelength will do so as well. But, as Austen Clark (forthcoming: Ch. 6) has pointed out, colour vision's testimony as to where differences lie tells vastly more reliably, and more usefully, about the objects that surround the organism. It attunes the organism to edges, places where one surface stops and another begins, that would be undetectable to black-and-white vision. It can steer a motor routine to reach for a banana that would otherwise remain indiscernible against the jungle background. It can signal to the organism that the same object extends beyond a narrow obstructing object both to the right and the left—and hence that a tail-like appendage is connected to a head with fangs bared. Finally, colour vision can render the organism 
sensitive to multi-dimensional complexes of shape and chromatic contrast, with the result that behaviour can be cued to the presence of bird wings with that pattern, or predator coats with those spots, across a wide variety of lighting conditions. So it can trigger the organism to do what it has learned to do, or has been hard wired to do, in the presence of just that other species. And note that the edges and contrast patterns which colour vision thus works by tracking are all features of objects out there around the organism, and not of the organism itself. Colour vision is not narcissistic — but more on this in a moment.

What about olfaction? If we generalize the points just made about colour vision, I think parallel points will appear plausible concerning olfaction as well. Colour vision works by signaling where objects in the environment begin and leave off and crop up again, both across space and time. It signals these things sometimes of individual objects, and sometimes of objects as embodiments of a kind—sometimes signaling, for example, "that kind of bird wing again". Olfaction, too, apparently does one of its jobs by signaling the reappearance of this or that individual conspecific animal-for in some species there are scent glands apparently selected precisely for enabling such reidentification. It is plausible that olfaction discharges other proper functions by signaling the presence of this or that kind, e.g. of plant or insect or prey. For in doing so it can trigger appropriate behaviour which natural selection has hard wired into the organism's repertoire, or can bring to bear information which the individual organism has learned from previous encounters with the kind (thus guiding behaviour now), or can even enable the acquisition of more such information (thus guiding future behaviour).

But it also is likely that olfaction sometimes works in a way colour vision typically does not. Olfaction may work by telling not just what is the same again and what different, in the objects that surround its owner, but by telling, of one such object, what state it is in. Surely part of what olfaction was selected for was triggering avoidance of foods that are rotten or of conspecifics that are ill. In these cases, no less than in those where olfaction signals bare sameness and difference, it works by signaling matters out there in the environing objects, not features of its host. The nose is no narcissist.

But perhaps the suggestion of narcissism cannot be dismissed merely by observing that olfactionor, for that matter, colour vision—gears its influence over behaviour to features and samenesses located out there in the objects. Perhaps both olfaction and colour vision work by signaling properties out there in 
the surrounding objects, but "response-dependent" properties. Perhaps, for example, the sensation in humans of red signals the presence of a dispositional property, namely the capacity to elicit in humans the sensation of red. In that case colour vision would still be in the interesting sense "narcissistic". The properties it signals would be anthropocentric, and strangely gerrymandered from a physical point of vie w. The signaling of such properties wold tell a lot more about us than about objects.

What I have maintained, however, is that the crucial question is how colour vision bestows selective advantage — what explains the fact that tugs and pulls which it administered (directly or indirectly) to the steering wheel of our ancestors' motor systems steered them, on the occasions decisive in evolution, in ways that helped them. That colour vision steered the motor systems towards or away from anthropocentric, gerrymandered reflectance properties does not explain why its steerings helped those ancestors. For there is no payoff in an organism's orienting itself well in relation to such properties. Even facing such properties squarely and saluting cannot $d o$ anything for (or against) the organism's physical or reproductive needs. Or, to be precise, if organisms did profit by having the direction of their motor routines attuned to the presence of the gerrymandered properties, they profited indirectly: just to the extent that, and just because, they thereby were attuned to edges and repeating contrast patterns genuinely there in the objects that surrounded them. Colour vision is not narcissistic in any sense.

Narcissism has a deservedly bad name, and I am glad to conclude that many of our sensory systems are innocent of the charge.

University of Connecticut, $U-54$

Storrs, CT 06269-2054, USA

elder@uconnvm.uconn.edu

\section{References}

Akins, K. 1996. What sensory signals are about. Journal of Philosophy 93: 337-72.

Clark, A. forthcoming. A Theory of Sentience. Oxford: Oxford Univ. Press.

Millikan, R. 1989. Biosemantics. Journal of Philosophy 86: 281-97.

Millikan, R. 1993. Truth rules, hoverflies, and the Kripke-Wittgenstein paradox. In her White Queen Psychology and Other Essays for Alice. Cambridge, Mass.: MIT Press. 
